\title{
La Educación Flexible: estrategia para la configuración de universidades virtuales en Colombia
}

Claudia-Rocío Benítez-Saza, Juan-Esteban Santamaría-Rodríguez y Scarlet Sotomayor-Tacuri

\section{RESUMEN}

El artículo revisa y reflexiona sobre la incursión e implementación de la educación flexible (EF) en la educación superior en Colombia como estrategia de formación de profesionales para la virtualización. Es decir, se identifican las fuerzas que han permitido generar una estrategia de poder/saber, y que hoy permite ir constituyendo un profesional flexible para la virtualización del trabajo, a través de una pesquisa de corte arqueológica-genealógica, en donde el uso de la arqueología permitirá ir al pasado, exactamente hasta dónde la EF hace su emergencia como estrategia de formación para la virtualización de los profesionales. Escudriñar la emergencia, remite exclusivamente a cuándo el discurso de "flexibilización" hace su aparición para la constitución de universidades virtuales en Colombia.

Palabras clave: educación flexible, universidades virtuales, análisis histórico, Colombia.

\section{Claudia-Rocío Benítez-Saza} Educativo y Social de la Universidad Pedagógica Nacional, Colombia. Adscrita como docente investigadora de la Universidad Distrital Francisco José de Caldas. Temas de investigación: pedagogía, tecnologías de la información y la comunicación, didáctica, currículo. ORCID: orcid.org/0000-0001-9470-5471.

\section{Juan-Esteban Santamaría-Rodríguez}

juanessantrax87@gmail.com Colombiano. Doctorando en Educación, Universidad Católica de Córdoba, Argentina; Magíster y Licenciado en Teología, Pontificia Universidad Javeriana, Colombia. Docente e investigador de la Facultad de Teología de la Universidad Santo Tomás, Colombia; investigador del Instituto Iberoamericano de La Haya para la Paz, los Derechos Humanos y la Justicia Internacional, Países Bajos; investigador adjunto del Centro Latinoamericano de Estudios en Epistemología Pedagógica, Cuba. Temas de investigación: pedagogías latinoamericanas, pedagogías críticas, investigación pedagógica, teologías latinoamericanas y teologías contextuales. ORCID: https://orcid.org/0000-0002-4632-4700.

\section{Scarlet Sotomayor-Tacuri}

Colombiana. Magister en Ciencias Sociales con mención en Género y Desarrollo, Facultad Latinoamericana de Ciencias Sociales, Ecuador. Especialista en Políticas públicas y justicia de género del Consejo Latinoamericano de Ciencias Sociales, Argentina; Antropóloga de la Universidad Icesi, Cali, Colombia; investigadora independiente. Temas de investigación: movimientos sociales, diversidades sexuales, género y educación. ORCID: https://orcid.org/0000-0002-6556-3600. 


\section{A Educação Flexível: estratégia para a configuração de universidades virtuais na Colômbia}

\section{RESUMO}

O artigo revisa e reflete sobre a incursão e implementação da educação flexível (EF) na educação superior na Colômbia como estratégia de formação de profissionais para a virtualização. Ou seja, se identificam as forças que têm permitido gerar uma estratégia de poder/saber, e que hoje permite ir constituindo um profissional flexível para a virtualização do trabalho, a través de uma pesquisa de cunho arqueológicagenealógica, onde o uso da arqueologia permitirá ir ao passado, exatamente até onde a EF teve sua emergência como estratégia de formação para a virtualização dos profissionais. Examinar a emergência, remete exclusivamente a quando o discurso de "flexibilização" faz sua aparição para a constituição de universidades virtuais na Colômbia.

Palavras chave: educação flexível, universidades virtuais, análise histórico, Colômbia.

\section{Flexible Education: strategy for the configuration of virtual universities in Colombia}

\section{ABSTRACT}

The article reviews and reflects on the incursion and implementation of flexible education (FE) in higher education in Colombia as a strategy for training professionals for working in virtual platforms. In other words, it identifies the forces that have made it possible to generate a strategy of power/knowledge, and which today make it possible to make up a flexible professional for the virtualization of work, through an archeological-genealogical investigation in which the use of archeology will make it possible to go back exactly to the point where the FE emerges as a training strategy for the virtualization of professionals. With "scrutinizing the emergence" the authors refer exclusively to when the discourse of "flexibilization" makes its appearance for the constitution of virtual universities in Colombia.

Key words: flexible education, virtual universities, historical analysis, Colombia. 


\section{Introducción}

La educación flexible (EF) se experimenta hace más de dos décadas con la reorganización de los componentes primordiales del sistema educativo en Colombia: enseñanza y aprendizaje (Díaz-Villa, 2002: 29-36; Salinas, 1998: 133-135; González, 2008: 3). En las prácticas educativas actuales, se sostiene la idea de que los estudiantes son el centro de la formación y son gestores de su aprendizaje; lo cual se debe, en parte, al auge de la modernización del siglo XXI que exige formarse con flexibilidad, actualización y permanentemente (Salinas, De Benito y Lizana, 2014: 150-153), tal como lo contempla el Ministerio de Educación Nacional (MEN) en el Plan Decenal de Educación 2016-2026:

\begin{abstract}
entendemos que la calidad en educación es una construcción multidimensional. Ésta solo es posible si desarrollamos simultánea e integralmente las dimensiones cognitiva, afectiva, social, comunicativa y práctica, de los colombianos y de la sociedad como un todo. Es, por tanto, una imagen que se construye con diferentes puntos de vista que convergen en ideas colectivamente creadas y aceptadas, que impulsan el cambio en la sociedad hacia el desarrollo humano integral y sostenido (MEN, 2017: 15).
\end{abstract}

En ese sentido, el modelo educativo flexible (Salinas, 2000: 10-14; 2002: 4-13), o sea, la reorganización de las técnicas que tienen lugar en las instituciones educativas, las prácticas pedagógicas en el aula y el currículo; constituyen los cambios que valen como fundación y renovación de los sistemas educativos. Para ello, rastreamos aquello que se funda en la transformación de la educación, en la ruptura de la enseñanza y la consistencia de formación de los profesionales, y cómo estas rupturas y discontinuidades son base para constituir sujetos de la virtualidad (Foucault, 2001).

En 2003, a través del documento denominado "La Revolución Educativa", el MEN propuso que dicha revolución significaría transformar la totalidad del sistema educativo en magnitud y pertinencia. Afirma, además, la exministra Cecilia Vélez: "debemos usar - e inventar si es preciso - métodos flexibles y creativos que nos permitan incorporar en el sistema educativo a todos los niños que aún no están en él” (MEN, 2003: 4). Entonces, la EF es un sistema que comprende reformas pedagógicas y didácticas en la escuela, justificadas en los campos de la política pública y la administración gubernamental (Díaz-Villa, 2002: 117-130).

El gobierno nacional colombiano impulsó la Revolución Educativa hace ya casi dos décadas. En ella se precisa una revolución de la rigidez a la flexibilidad, impulsando con ello retos como: 1) ampliación de cobertura, 2) mejoramiento continuo de la calidad de la educación a través de la aplicación de los estándares mínimos para todos los niveles del sistema educativo, 3) formulación de planes de mejoramiento institucional, 4) desarrollo de competencias que le permitan a los niños y jóvenes enfrentarse a los retos y exigencias de la sociedad de la información y el conocimiento, y 5) diseño de herramientas que aseguren la eficiencia del sector a través del fortalecimiento del sistema de información educativo y la modernización de las entidades territoriales (Veléz, 2002).

Ahora bien, la transformación de la educación tradicional (que se presenta como obsoleta) a una educación mediada por las tecnologías de la información y la comunicación (TIC), para resolver tales dificultades desde el ámbito gubernamental, como por ejemplo el Plan de Gobierno, implica una "Educación para Todos", trayendo consigo una estrategia de flexibilización de la formación que describe el Ministerio de Educación Nacional, en el año 2014, como:

propuestas de educación formal que permiten atender a poblaciones diversas o en condiciones de vulnerabilidad, que presentan dificultades para participar en la oferta educativa tradicional. Estos modelos se 
caracterizan por contar con una propuesta conceptual de carácter pedagógico y didáctico, coherente entre sí, que responde a las condiciones particulares y necesidades de la población a la que se dirigen (MEN, 2014).

No obstante, para las instituciones educativas no ha sido claro qué es lo que implica hablar de EF, sobre todo, a través de la utilización de las TIC, pues por un lado, la asimilación de estas tecnologías a la vida cotidiana se lleva a cabo de manera tan natural, que parecería que todos comprendemos perfectamente en qué consiste su uso para la flexibilización de la educación; sin embargo, por otro lado, cabe preguntarse: ¿qué implican los discursos que legitiman el uso de modelos educativos flexibles? ¿por qué dichos discursos promueven las TIC como bandera para la EF? Para empezar a responder estos interrogantes, creemos necesario precisar aquello que en la actualidad se denomina EF y "virtualidad", lo que nos permitirá enseguida analizar la forma en que esta noción se ha legitimado en el campo educativo.

\section{Educación flexible: historicidad en Colombia}

El método (arqueológico y genealógico) es un entramado de análisis de discursos y de prácticas, el cual se pregunta por las reglas generales de las relaciones entre los enunciados que configuran un saber determinado, en este caso la "Educación flexible" en una época dada. Así pues, es una analítica de los saberes institucionalizados que describe las reglas para los sujetos y las instituciones sobre los discursos de un saber específico:

Este enfoque para analizar las prácticas de saber acoge los documentos, no tanto como fuente, sino como Registro de prácticas; el documento no es una fuente para hacerla hablar desde otra discursividad, es un registro donde ya se ha hablado desde un ejercicio de saber, cruzado por instituciones, discursos, sujetos y prácticas diferentes; de ahí que se asuma el documento, visto desde su discurso, como un tejido relacional que permite una reescritura metódica de lo dicho (Zuluaga, 1987: 18).

Así, el rastreo de los enunciados de la EF nos lleva a los últimos 60 años (Maldonado, 2011: 45-58. 76-108), pues tanto la escuela como los profesores se han visto colmados por los diferentes procesos novedosos provenientes de los campos de saber de la tecnología, tecnología educativa, radio educativa, televisión educativa, informática educativa, y en las últimas tres décadas la invasión provocada por las TIC. Estos procesos tecnológicos que bombardean a los profesores y estudiantes reorganizan los componentes primordiales del sistema educativo del país: la duplicidad de la enseñanza y el aprendizaje.

Las concepciones pedagógicas pasan de pensarse para la enseñanza a estructurarse en el aprendizaje, el auto-aprendizaje y el aprendizaje colaborativo. Ya desde el gobierno nacional se recomiendan pedagogías con finalidades, sólo para que los estudiantes logren aprendizajes que demanda el siglo XXI. Así, el MEN (2007) determina que la formación de profesores es la fundamentación pedagógica, epistemológica y didáctica del uso de la tecnología, sin la cual es imposible que la educación cumpla los objetivos propuestos, pues la incorporación que se pretende no es meramente instrumental, tal como lo plantea Claudio Rama (2012: 17) cuando afirma que:

Desde la palabra hacia las imágenes, desde lo presencial a lo virtual, desde lo colectivo hacia lo individual, desde el texto al hipertexto, desde lo escrito a lo multicomunicacional, desde lo estático a lo móvil, permiten desarrollar nuevas pedagogías y estrategias que, a su vez, son parte de un nuevo paradigma emergente que promueve la construcción de una práctica digital a través de la Web y de multiplicidad de aplicaciones y recursos digitales de aprendizaje con base en modelos semi-presenciales o totalmente virtuales. 
El impacto de un modelo educativo integral y flexible, que se explica como la organización de las técnicas que tienen lugar en las instituciones educativas colombianas, desde las prácticas pedagógicas en el aula hasta el currículo, constituyen la capacitación de profesionales para la virtualización educativa. De esta manera, persisten los procesos de gestión y administración en formación en las TIC. Así pues, la $\mathrm{EF}$ es el componente tecnológico fundamental para desarrollar los procesos de virtualización de la educación (Cabero, 2004: 13-15) en donde los discursos sobre la incorporación de las TIC en las prácticas de formación son pronunciados por diversos actores: el MEN, las instituciones de educación superior con programas de formación de profesionales a distancia y virtuales, las empresas de comunicaciones, e inclusive por los propios estudiantes universitarios. ${ }^{1}$

Lo que se ha denominado en la política pública educativa como modelo flexible de aprendizaje o de inclusión educativa, converge en la educación integral, que se planeó para conducir todos los programas y proyectos de las dos últimas décadas en Colombia. La diversidad de etiquetas para nombrar un sistema educativo que se está flexibilizando y actualizando constantemente, confunde a los docentes y estudiantes en las prácticas de formación, especialmente, por dos razones. La primera es que la educación superior se concretiza en la flexibilización de los currículos de formación en cada institución educativa, es decir un cambio gradual y sistemático en las formas de aprender y de enseñar (MEN, 2007); la segunda razón es que intentar una conceptualización de un enfoque orientado al desarrollo de competencias docentes es complejo y está en debate constante en las instituciones educativas.
El actual sistema educativo colombiano se ha sometido a una serie de diagnósticos y problematizaciones $^{2}$ que, en consecuencia, ha llevado al gobierno nacional a plantearse una revolución educativa con el fin de hacer posibles las metas educativas contempladas en el Plan Decenal de Educación 2016-2026, "El camino hacia la calidad y la equidad" (MEN, 2017), y sobre las cuales quiere responder a los supuestos problemas de la educación: 1) derecho constitucional a la educación; 2) sistema educativo articulado, participativo y descentralizado; 3) diseño de lineamientos curriculares generales, pertinentes y flexibles de acuerdo con las necesidades contextuales; 4) generación de políticas públicas para la formación de educadores; 5) transformación del paradigma educativo clásico; 6) usos pertinentes, pedagógicos y generalizados de las nuevas y diversas tecnologías para el apoyo de los procesos de enseñanza y aprendizaje; 7) construcción de sociedades en paz desde la equidad, inclusión, ética y equidad de género; 8) desarrollo prioritario a la población rural desde los procesos educativos; 9) relevancia del papel del Estado en el sistema educativo desde su participación a través del PIB, y 10) fortalecimiento de los procesos investigativos orientados a la generación de conocimientos en los niveles educativos estipulados por el MEN en el país (MEN, 2017: 39-66).

Durante los últimos 25 años algunas propuestas de
cambio han tenido una mayor aceptación en gran
parte de los países de la región. El telón de fondo de la
mayoría de ellas ha sido la vinculación de los objetivos
de calidad con la eficiencia en la gestión de los recur-
sos económicos, con la descentralización de las polí-
ticas educativas y con la evaluación estandarizada de
los logros académicos de los alumnos (OEI, 2010:31).

\footnotetext{
${ }^{1}$ Para el proceso de acreditación de alta calidad las instituciones de educación superior en Colombia y sobre todo, de sus programas académicos, en más de un 80\% son promovidas distintas prácticas asociadas al uso de las TIC como recurso pedagógico. Éste es un factor que busca garantizar el alto impacto que ostentan tales tecnologías, así como asegurar el proceso para la descentralización de los procesos educativos desde experiencias ubicuas, bajo criterios sincrónicos y asincrónicos (Galasso, 2018: 92-94; Araújo y Oliveira, 2018: 3-5).

${ }^{2}$ La problematización obedece a una transformación del poder, en el que se pretende fracturar un discurso. Para Foucault, "problematización es el conjunto de las prácticas discursivas (o no discursivas) que hacen que algo entre en el juego de lo verdadero y de lo falso y lo constituye como objeto para el pensamiento (reflexión moral, conocimiento científico, análisis político)" (Foucault, 1999: 371).
} 
Entonces, la emergencia de la EF consiste en actualizar y mejorar los planes de estudio, los currículos, así como la instrumentación de diversos programas de formación para los estudiantes. Es decir, la EF está encaminada a disminuir las tasas de fracaso y rezago escolar, además de mejorar la calidad de la educación (MEN, 2007). Esto significa, según el MEN, que la revolución educativa es calidad y toda propuesta, reforma o modelo debe construirse con este fin (MEN, 2017: 23-25).

En las instituciones educativas colombianas se promueven y gestionan proyectos de modelo integral y flexible. Éstos afrontan el desafío de nuevas formas de organización y direccionamiento, de formación y estructura, demandando cambios disímiles en las aulas de clase. Ahora bien, los docentes son gestores de estos procesos, quienes regularmente no tienen precisión, puesto que el modelo pedagógico que orienta su trabajo hacia el cumplimiento de la actualización y flexibilización de la enseñanza, se justifica en la meta del mejoramiento de la calidad educativa a modo estandarizado. El MEN, en el marco de la política de calidad, y tomando como base la experiencia en la aplicación de metodologías flexibles, presentó a la comunidad un portafolio de modelos educativos al respecto:

Los modelos flexibles tienen su sustento conceptual en las características y necesidades presentadas por la población a la cual busca atender y se apoya en tecnologías y materiales educativos propios. A través de la implementación de los mismos, se busca generar los mecanismos necesarios para que, reconociendo sus especificidades, se les brinde a estas poblaciones oportunidades de acceder, permanecer y promocionarse en condiciones apropiadas de calidad, pertinencia, eficiencia y equidad (MEN, 2006: 7).
Los procesos pedagógicos que promueven la EF están cargados de teorías, por mencionar algunas como: aprendizaje colaborativo, auto-aprendizaje y las competencias (Silva, 2014: 12; Castaño-Garrido y Themistokleous, 2018: 144-148). En este sentido, las reformas en la educación buscan la integración entre las TIC, la gestión y las competencias para la capacitación de los niños, jóvenes y docentes en nuevos entornos de aprendizaje y cooperación (UNESCO, 2006). Los procesos pedagógicos integrales, con el fin de responder a las necesidades locales, regionales y mundiales, deben innovarse, flexibilizarse y gestionarse, según este mismo organismo. En conclusión, el modelo educativo flexible es una de las recomendaciones de la OCDE toda vez que busca "mejorar la calidad de los modelos flexibles y la Educación para el Trabajo y el Desarrollo Humano para facilitar la incorporación a la economía formal" (OCDE, 2016: 11). En este sentido, las características de la EF se afianzan a partir de: "La inclusión y la integración de las TIC que se encuentran ligadas con políticas de equidad y por tanto no se trata de correr tras la novedad sino de aprovechar las TIC como ventana de oportunidad para incorporar cambios pedagógicos que favorezcan el mejoramiento del sistema educativo" (UNESCO, 2006:11).

Es allí donde los docentes se enfrentan a reformas educativas constantes, con características fundamentales como modelos en función de la innovación, para el uso de las TIC y para desarrollar competencias propias de la sociedad de la información y el conocimiento (SIC). Así pues, esta consideración acerca del surgimiento de la EF a partir de la actualización permanente y la informática educativa, ha autorizado recientemente a Mario Díaz-Villa (2002: 14)3 a afirmar que a nivel de la educación superior "significaría la posibilidad de modificación de las prácticas existentes y la articulación compleja, dinámica y fluyente de

\footnotetext{
${ }^{3}$ Doctor de la University of London en Sociología de la Educación, experto en el campo de la pedagogía que promueve la educación flexible como consultor para el Ministerio de Educación Nacional en Colombia.
} 
procesos de apertura, innovación y cambio", la cual se consolida en la Universidad Nacional de Colombia en 1999 y, posteriormente, en la Universidad de los Andes en 2000 con restructuraciones de sus proyectos educativos institucionales. Sin embargo, estas afirmaciones sobre el surgimiento de la EF no son tan precisas como suponen los autores mencionados. De esta manera, a continuación, veremos que hay una emergencia de EF que se constituye en otros campos de poder.

\section{Emergencia de la EF en Colombia}

Las fuerzas de los acontecimientos no se posicionan en la historia con una línea cronológica, más bien comprendemos que la historia es la del pensamiento y que, en este caso, estamos en la búsqueda de la emergencia de la EF. Según propone Nietzsche, esta noción de historia nos permite precisar en qué momento aparece esta nueva fuerza en ella. Así pues, se trata de la emergencia (entstehung), entendida como "el punto de surgimiento. Es el principio y la ley singular de una aparición" (Foucault, 1979/1971: 15) en ciertas condiciones específicas: "La emergencia es pues, la entrada en escena de las fuerzas; es su irrupción, el movimiento de golpe por el que saltan de las bambalinas al teatro, cada una con el vigor y la juventud que le es propia. Lo que Nietzsche llama la "Entstehungsherd". [...] Nadie es pues responsable de una emergencia, nadie puede vanagloriarse; ésta se produce siempre en el intersticio" (ibid.: 15-16).

Los procesos disciplinares (adelantos y retrocesos) van compaginados con la incursión de diferentes teorías y corrientes de pensamiento (paradigmas) sobre el sujeto. Por eso, la EF configura y es configurada, como cualquier forma de conocimiento, a la vez por los paradigmas, los lugares de los sujetos y la conformación de los saberes. En este sentido, es posible un desplazamiento hasta mediados de 1966, momento en que se operan algunas recomendaciones del Comité de la OIT y la UNESCO en la formación para la educación superior: "Los Estados Miembros y a los proveedores de formación para el personal docente para que utilicen un enfoque integrado que permita abordar la preparación de los docentes bajo la óptica de que el aprendizaje es un proceso continuo de la formación, la inducción, el desarrollo profesional del personal docente y el estudio por sus propios medios" (OIT-UNESCO, 2008: 5).

Las comisiones hacen alusión a la ruptura de la enseñanza-aprendizaje, en el que las reformas educativas adoptarán los procesos centrados en el aprendizaje como un foco de la educación ubicua y continua (Galasso, 2018). En Colombia, esto se reglamenta en 1982 como "Educación Abierta y a Distancia" con el Decreto 2412. Allí se propone una educación superior abierta que permita a cada alumno ser agente de su propio perfeccionamiento, colocando con ello en funcionamiento las exigencias de la virtualización de la formación mediante el diseño flexible (Silvio, 2000).

Ahora bien, la perspectiva que aquí nos interesa es la que define la EF como aquella que facilita el acceso y la permanencia en la formación a través de plataformas y programas a distancia. Es así como los modelos educativos flexibles desarrollan procesos de aprendizaje que se ajustan a las necesidades de los niños, niñas, adolescentes y jóvenes, especialmente, en términos de tiempo y ubicación de acuerdo con programas como "Colombia aprende" del MEN. Además, nos interesa la virtualidad como "un conjunto de nuevas relaciones comunicacionales, intercambio de datos, información o conocimiento que se inaugura en torno a las nuevas tecnologías de la información, y de forma más específica, en torno a internet" (Galzacorta, 2008: 310). Entonces, nuestro rastreo lleva a preguntarnos: ¿por qué se alinean las fuerzas de la formación virtual y la EF? ¿qué función tiene la EF en la constitución de sujetos para la virtualización?

Las TIC condicionan este proceso de virtualidad y flexibilización de la educación superior, en tanto que brindan "la posibilidad de relacionarse a distancia sin necesidad de estar en un mismo local o 
territorio" (Echeverría, 2000: 16). En esta perspectiva aparecerán universidades, clases, libros, bibliotecas que pueblan el campo de la educación virtual y su fluidez. Sólo situando la EF en el marco de las TIC tiene sentido preguntarse por el nuevo lugar y la nueva función de los profesionales. Para expertos como Silvio (1998), en la educación superior la virtualización puede comprender la representación de procesos y objetos asociados a actividades de enseñanza y aprendizaje mediados por internet que flexibilizan la interacción con cursos electrónicos, inscribirse en un curso, consultar documentos en una biblioteca electrónica, comunicarse con estudiantes y profesores, entre otros. Ahora bien, los discursos de la EF pueden llegar a consolidar la formación virtual para configurar sujetos operarios de las TIC o para su emancipación, lo cual genera una tensión dialéctica necesaria de resolver de acuerdo con su contextualización en el entramado neoliberal y globalizante en el cual está situada la educación superior hoy (DíazVilla, 2011: 10-15).

\section{Discursos de la virtualización: fluidez y actualización del trabajo}

$\mathrm{Al}$ analizar la denominada SIC, categoría propuesta por Castells (1997) y que alude a la transformación social constituida por el material del paradigma de las TIC, en la que uno de sus rasgos es la flexibilización de los procesos, las instituciones y las organizaciones hacia la modificación de todos sus componentes, hallamos una referencia a las sociedades de control que sustituyen a las disciplinarias: "Las disciplinas entraron en crisis en provecho de nuevas fuerzas que se iban produciendo lentamente, y que se precipitaron después de la segunda Guerra Mundial: las sociedades disciplinarias son nuestro pasado inmediato, lo que estamos dejando de ser" (Deleuze, 2006: 278).

De igual manera, Deleuze (2006) nos advierte que estamos entrando a las sociedades de control, las cuales funcionan mediante un control continuo y una comunicación instantánea. El asunto es que las instituciones están en crisis; es decir, se está instaurando con cierta lógica un nuevo tipo de sanción, de educación, de vigilancia (Deleuze, 2006). La referencia al lugar educativo, señala la presunción de que la educación deje de ser paulatinamente un alojamiento hermético (lugar de los cuerpos dóciles en los niveles conocidos de formación) y que esos lugares de alojamiento concedan espacio "en provecho de una terrible formación permanente, un control continuo que se ejercerá sobre el obrero-estudiante de secundaria o sobre el directivo-universitario. Se nos quiere hacer creer en una reforma educativa, pero se trata de una liquidación. En un régimen de control, nada se termina nunca" (Deleuze 2006: 273).

La EF es la fuerza que debilita las sociedades disciplinarias y cede terreno a la formación virtual, dispositivo de control de los sujetos y la fluidez de la sociedad moderna: "La rigidez del orden es el artefacto y el sedimento de la libertad de los agentes humanos. Esa rigidez es el producto general de "perder los frenos": de la desregulación, la liberalización, la "flexibilización", la creciente fluidez, la liberación de los mercados financiero, laboral e inmobiliario, la disminución de las cargas impositivas, etcétera" (Bauman, 2004: 4).

La educación superior no es ajena al proceso de fluidez, puesto que las TIC han configurado una nueva institución del conocimiento y tienen un vínculo con la virtualización como función de la actualización de métodos educativos cuyas posibilidades se ven ampliadas con su uso. Además, es la tendencia económica y cultural que colma el ámbito de la educación escolar.

La educación superior tiene un conjunto de prescripciones que se refieren a los discursos aceptados de la flexibilización a través del cambio constante, la actualización y restructuración por medio de los docentes que se apropian de las TIC en sus modelos de aprendizaje. Por ello se afirma que "la integración exitosa de las TIC a la enseñanza depende del papel que jueguen los docentes en la incorporación de ellas 
al aula de clase, de sus habilidades, creencias y actitudes frente a estas tecnologías" (Espinosa, Aranzazu y Restrepo, 2014: 154). Esto, en palabras de Lyotard (1991: 8), denota aquello "que no impide que, en los flujos de dinero, unos sirvan para decidir mientras que los otros sólo sirvan para adquirir". La fluidez del conocimiento presenta al profesional como un sujeto proveedor, pero al profesional fuera de la fluidez como un usuario. Así entonces, nuestra hipótesis es que enfrentamos, por medio de la EF, la lógica de una sociedad tecnologizada que camina sobre prácticas tecnócratas muy distantes a la revitalización del acto educativo en su divergencia, pluralidad y criticidad (Mejía, 2004).

La flexibilización educativa se normaliza, dado que las oleadas tecnológicas justifican la transformación masiva en la manera como se educan las personas y se transmiten los saberes. Como lo afirma Rama (2012: 22), "el mundo del trabajo se articula con ambientes digitales y se incorpora la informática en tanto herramienta del trabajo para incorporar valor agregado de conocimientos en forma digital".

Parada (2010) propone para las comunidades académicas las tecnologías digitales como el camino para mejorar la educación. De ahí que, en las prácticas universitarias, desde 2010 los docentes requieran hacer un uso cotidiano del computador para mejorar sus competencias en TIC y, con ello, lograr impactar en el proceso de enseñanza y aprendizaje remoto. Así mismo, los materiales digitales en los planes de estudio son exigidos junto con la formación en las competencias digitales a los docentes, con legitimizaciones como facilitar, optimizar y permanecer en conexión. Es decir, que para el caso de las universidades colombianas con modalidades a distancia y virtuales se aumentan sus dinámicas, creando nuevas formas de docentes, estudiantes y, en general, profesionales flexibles (Arboleda y Rama, 2013). Esto, sin lugar a dudas, es la adaptación al mundo en movimiento y la diferenciación de lo real al simulacro (Virilio, 2003), en donde el sujeto da el paso de la comunicación a la digitalización, base de la formación contemporánea que no tiene límite y re-presenta la velocidad de millones de bits de información, conllevando a formas de EF diversas, y por qué no, diametralmente opuestas.

\section{Conclusiones}

El sistema universitario en Colombia, en la última década, ha transformado sus dinámicas en los programas que se ofertan como globales de forma automatizada a través de la red (Rama, 2012). De igual manera, la educación superior se reforma a través de políticas de acreditación de alta calidad que les exigen flexibilización e incorporación de las TIC a las instituciones de educación superior (MEN, 2018, Decreto $\mathrm{n}^{\circ}$ 1280, art. 2.5.3.2.2.2.3). Así, por ejemplo, la Corporación Universitaria Minuto de Dios-UNIMINUTO,${ }^{4}$ ha desarrollado nuevas modalidades que ofertan educación superior para quienes, se supone, no tienen oportunidades de acceder a ella, a través de un modelo innovador, integral y flexible (UNIMINUTO, 2017). Esto significa que la formación de profesionales se constituye a partir de la flexibilización y para la virtualización, puesto que "el adiestramiento en el manejo de las nuevas tecnologías no es sólo un requerimiento laboral, sino que son competencias crecientemente necesarias de la vida en sociedad en un proceso aluvional de digitalización” (Rama, 2012: 18).

La descripción histórica que se ha realizado, el análisis de las formas que la educación flexible ha asumido y a través de las cuales se ha instaurado, las condiciones de posibilidad para la emergencia de la educación flexible, primero en las universidades y después en todo el campo institucional, son

\footnotetext{
${ }^{4}$ Más de cien universidades en Colombia en busca de acreditación de alta calidad han prescrito el enunciado de flexibilización en sus programas. Este requisito sólo puede entenderse si se comprende la actualización de los procesos pedagógicos de las instituciones de educación superior en el país de acuerdo con la normativa vigente y, por otro lado, como estrategia para su permanencia en el mercado académico y educativo a nivel nacional.
} 
características propias de una virtualización de las funciones de los individuos que buscan: "generar una nueva cultura que permita superar las tradicionales prácticas académicas e institucionales caracterizadas por la rigidez y el aislamiento, e impulsar nuevas formas de trabajo, que puedan definirse por flexibilidad, la pertinencia, el trabajo colaborativo, la autonomía, y la vinculación de la educación superior con otras instituciones" (Díaz-Villa, 2002: 10).

El análisis de la estrategia de saber/poder para el establecimiento de la flexibilización en la educación superior en Colombia visibiliza un entramado de discursos legitimadores de la llamada SIC. A su vez, el auge de las TIC y la crisis académico-administrativa de la universidad son las condiciones que permiten la emergencia del discurso de la EF. Sin lugar a duda, esta flexibilidad educativa se ha constituido en una estrategia de la sociedad moderna para hacer sujetos productivos en su aprendizaje, así como usuarios del conocimiento (Gutiérrez, Ulloa y Simancas, 2018), no sin advertir del riesgo que esto supone en cuanto a consumo de conocimiento, se refiere en el marco de las lógicas capitalistas cognitivas que sustentan estas tendencias pedagógicas (Mejía, 2010: 61-63).

\section{Referencias}

Araujo, D. y S. Oliveira (2018), "Reflexão sobre o contexto da educação a distância e a importância da ação do designer instrucional no ambiente virtual de aprendizagem", Anais CIET: EnPED, pp. 1-12, <https:// goo.gl/kgDtff> [Consulta: marzo de 2018].

Arbólela, N. y C. Rama (2013), La educación superior: a distancia y virtual en Colombia: nuevas realidades, Bogotá, Asociación Colombiana de Instituciones de Educación Superior con Programas a Distancia y Virtual: VIRTUAL EDUCA, < https://bit.ly/2RaaATW> [Consulta: enero de 2018].

Bauman, Z. (2004), Modernidad líquida, Buenos Aires, Fondo de Cultura Económica.
El análisis también pone en evidencia que la educación virtual refuerza la necesidad de que los sujetos estén interconectados y hagan parte de la globalización y la innovación, siendo ambos enunciados hegemónicos de productividad en el mundo actual. Si bien los discursos de oportunidad o desventaja de las universidades respecto del uso de TIC cuestionan las prácticas tradicionales de educación, también sitúan una dialéctica sobre su sentido en tensión con la masificación de las mediaciones pedagógicas que caminan hacia el capitalismo cognitivo y el consumo de conocimientos necesarios para la reproducción de técnicas concretas encaminadas a la producción y el desarrollismo económico.

Así entonces, tomamos un dispositivo concreto: universidades virtuales, para identificar el diagrama de la sociedad que se flexibiliza hacia un campo social con productores de conocimiento fluido. Sin embargo, frente a la sujeción de las universidades a la fluidez virtual y digital, estamos en la búsqueda de un "afuera" que se resiste: ¿qué tipo de institución se ha resistido a este régimen de tecnologización virtual que la diluye, lejos del ejercicio de la formación?

Cabero, J. (2004), "Las TIC como elementos para la flexibilización de los espacios educativos: retos y preocupaciones", Comunicación y Pedagogía, núm. 194, pp. 13-19, <https://bit.ly/2LupR0f> [Consulta: febrero de 2018].

Castaño-Garrido, C. y S. Themistokleous, S. (2018), "De la revolución del software a la del hardware en educación superior", RIED. Revista Iberoamericana de Educación a Distancia, vol. 21, núm. 1, pp. 135-153, <https:// bit.ly/2rVHwoH>, DOI: https://doi.org/10.5944/ ried.21.1.18823> [Consulta: junio de 2018].

Castells, M. (1997), La Sociedad Red, Madrid, Alianza Editorial. 
Corporacion Universitaria Minuto de Dios (UNIMINUTO) (2018), Acerca de la UNIMINUTO, Bogotá, Corporación Universitaria Minuto de Dios-UNIMINUTO, <https:/ / bit.ly/2AeoNcE $>$ [Consulta: febrero de 2018].

Deleuze, G. (2006), Conversaciones, 4, Valencia, Pre-Textos. Díaz-Villa, M. (2011), "Los discursos sobre la flexibilidad y las competencias en la educación superior", Pedagogía y Saberes, núm. 35, pp. 9-24, <https: //bit.ly/2Rk53y6> DOI: <https://doi.org/10.17227/01212494.35p ys9.24> [Consulta: mayo de 2018].

Díaz-Villa, M. (2002), Flexibilidad y educación superior en Colombia, Bogotá, Instituto Colombiano para el Fomento y Desarrollo de la Educación Superior ICFES, <https://bit.ly/2BBjoMw> [Consulta: marzo de 2018].

Echeverría, J. (2000), Un mundo virtual, Barcelona, Nuevas Ediciones de Bolsillo.

Foucault, M. (2001), La arqueología del saber, Buenos Aires, Siglo XXI Editores.

Foucault, M. (1999), Estética, ética y hermenéutica, Barcelona, Paidós.

Foucault, M. (1979/1971), "Nietzsche, la genealogía, la historia", en M. Foucault (ed.), Microfisica del poder $\left[2^{\circ}\right.$ Edición], Madrid, La Piqueta, pp. 7-29.

Galasso, B. (2018), "Aprendizagem ubíqua: o espaço online como lócus de realização educacional", Interfaces Científicas, vol. 6, núm. 3, pp. 85-96, <https://goo.gl/ L6C6Pu>, DOI: <http://dx.doi.org/10.17564/23163828.2018v6n3p85-96> [Consulta: noviembre de 2018].

Galzacorta, Í. (2008), "Leyendo lo virtual. Anotaciones en torno a la virtualidad de la escritura", Ontology Studies, núm. 8, pp. 307-316, <https://bit.ly/2GysyuT> [Consulta: enero de 2018].

González, J. (2008), "TIC y la transformación de la práctica educativa en el contexto de las sociedades del conocimiento", RUSC. Universities and Knowledge Society Fournal, vol. 5, núm. 2, pp. 1-8, <https://bit. ly/2GBwbEJ> DOI: <http://dx.doi.org/10.7238/ rusc.v5i2.330> [Consulta: julio de 2018].

Gutiérrez, M., H. Ulloa e I. Simancas (2018), "El docente en la educación y las TIC", Revista Educateconciencia, vol.
18, núm. 19, pp. 92-111, <https://bit.ly/2ESRujt> [Consulta: enero de 2018].

Lyotard, J. (1991), La condición postmoderna. Informe sobre el saber, Buenos Aires, Editorial R.E.I.

Maldonado, M. (2011), Pedagogías críticas. Europa, América Latina, Norteamérica, Bogotá, Editorial Magisterio.

Mejía, M. (2010), "Las pedagogías críticas en tiempos de capitalismo cognitivo. Cartografiando las resistencias en educación", Aletheia. Revista de desarrollo humano, educativo y social contemporáneo, vol. 2, núm. 2, pp. 58-101, <https:// bit.ly/2QVfKb7> [Consulta: marzo de 2018].

Mejía, M. (2004), "La tecnología, la(s) cultura(s) tecnológica(s) y la educación popular en tiempos de globalización", Polis. Revista Académica. Universidad Bolivariana, vol. 2, núm. 7, pp. 1-42, <https://bit. ly/2Slf1wp> [Consulta: abril de 2018].

Ministerio de Educación Nacional (MEN) (2018), Decreto número 1280 de 2018 (25 de julio de 2018), <https://bit. ly/2Oj1B2l> [Consulta: septiembre de 2018].

MEN (2017), Plan Decenal de Educación 2016-2026. "El camino hacia la calidad y la equidad", <https://bit. ly/2phXxDW> [Consulta: abril de 2018].

MEN (2014), Modelos educativos flexibles actualizados, <https://bit.ly/2QO4jlc> [Consulta: abril de 2018].

MEN (2006), Portafolio de modelos educativos, <https://bit. ly/2BEW0xP> [Consulta: abril de 2018].

MEN (2003), La Revolución Educativa. Plan sectorial 20022006, <https://bit.ly/2EQqCzL> [Consulta: abril de 2018].

MEN (1982), Decreto número 2412 de 1982 (Agosto 19), <https://bit.ly/2SmDm4K> [Consulta: abril de 2018].

Organización de Estados Iberoamericanos para la Educación, la Ciencia y la Cultura (OEI) (2010), Metas educativas 2021: La educación que queremos para la generación de los bicentenarios, Madrid, OEI, <https://bit. ly/2LwZzcV> [Consulta: julio de 2018].

OCDE (2016), Educación en Colombia. Aspectos destacados, Bogotá, OCDE, <https://bit.ly/2el9VAP> [Consulta: mayo de 2018].

Parada, D. (ed.) (2010), 7 claves para incorporar tecnología digital al proceso educativo. Experiencias y recomendaciones, Santiago 
de Chile, Fundación País Digital, <https://bit. ly/2LA8njh> [Consulta: septiembre de 2018].

Rama, C. (2012), La reforma de la virtualización de la universidad: el nacimiento de la educación digital, México, UDG VIRTUAL, $<$ https://bit.ly/2Snxb0j> [Consulta: junio de 2018].

Rodríguez, H., L. Restrepo y D. Aranzazu (2014), "Alfabetización informática y uso de sistemas de gestión del aprendizaje (LMS) en la docencia universitaria", Revista de Educación Superior, vol. 43, núm. 171, pp. 139159, <https://bit.ly/2AjmEfJ>, DOI: <https://doi. org/10.1016/j.resu.2015.03.004> [Consulta: agosto de 2018].

Salinas, J. (2002), "Modelos flexibles como respuesta de las universidades a la sociedad de la información", Acción Pedagógica, vol. 11, núm. 1, pp. 4-13, <https:// bit.ly/2Lw5MHc> [Consulta: junio de 2018].

Salinas, J. (2000), "Campus Extens: un modelo de formación flexible en entornos virtuales", en III Congreso Internacional de Comunicación, Tecnología y Educación. Redes multimedia y Diseños virtuales, Ovideo, España, <https:/ / bit.ly/2Ctvt8q> [Consulta: octubre de 2018].

Salinas, J. (1998), "El rol del profesorado universitario ante los cambios de la era digital", Agenda Académica, vol. 5, núm. 1, pp. 131-141, <https://bit.ly/2EDrg3d> [Consulta: marzo de 2018].

Salinas, J., B. de Benito y A. Lizana (2014), "Competencias docentes para los nuevos escenarios de aprendizaje", Revista Interuniversitaria de Formación del Profesorado, vol. 79, núm. 28.1, pp. 145-163, <https://bit.ly/2EHC5SQ> [Consulta: septiembre de 2017].

Silva, J. y M. Romero (2014), "La virtualidad una oportunidad para innovar en educación: un modelo para el diseño de entornos virtuales de aprendizaje", Didasc@alia: Didáctica y Educación, núm. 5, pp. 1-23, $<$ https://bit.ly/2SlplEJ> [Consulta: julio de 2018].

Silvio, J. (2000), La virtualización de la universidad: ¿Cómo transformar la educación superior con la tecnología?, Caracas, UNESCO, <https://bit.ly/2HLFRM2> [Consulta: febrero de 2018].

Silvio, J. (1998), "La virtualización de la educación superior: alcances, posibilidades y limitaciones", Revista Educación Superior y Sociedad, vol. 9, núm. 1, pp. 27-50, $<$ https:/ /bit.ly/2ENK6F0> [Consulta: abril de 2017].

UNESCO (2006), La integración de las tecnologías de la información y la comunicación en los sistemas educativos, Buenos Aires, UNESCO, < https://bit.ly/2GxA0dT> [Consulta: junio de 2017].

UNESCO/OIT (2008), Recomendaciones de la OIT y la UNESCO relativas a la formación docente (1966) y Recomendación de la UNESCO relativa a la condición del personal docente de enseñanza superior (1997), París, UNESCO, $<$ https:/ /bit.ly/2Shf6Rv> [Consulta: marzo de 2017].

Virilio, P. (2003), Arte del motor: aceleración y realidad virtual, Buenos Aires, Manantial.

Zuluaga, O. (1987), Pedagogía e historia: la historicidad de la pedagogía, Colombia, Siglo del Hombre.

Cómo citar este artículo:

Benítez-Saza, Claudia-Rocío, Juan-Esteban Santamaría-Rodríguez y Scarlet Sotomayor-Tacuri (2020), “La Educación Flexible: estrategia para la configuración de universidades virtuales en Colombia”, Revista Iberoamericana de Educación Superior (RIES), vol. XI, núm. 31, pp. 118-129, DOI: https://doi.org/10.22201/iisue.20072872e.2020.31.709 [Consulta: fecha de última consulta]. 